\title{
Índice de Mannheim y mortalidad en sepsis abdominal
}

\author{
Index of Mannheim and mortality in sepsis abdominal
}

\author{
Luis G. González-Pérez', Yazmín Sánchez-Delgado², Ansony R. Godínez-Vida2*, \\ José F. Cruz- Manzano², Gabriela E. Gutiérrez-Uvalle y Noé I. Gracida-Mancilla ${ }^{1}$ \\ ${ }^{1}$ Clínica de Sepsis, Servicio de Cirugía General; ${ }^{2}$ Servicio de Cirugía General. Hospital General de México Dr. Eduardo Liceaga, Ciudad de México, \\ México
}

\section{Resumen}

Introducción: La sepsis abdominal representa la respuesta inflamatoria peritoneal ante la invasión microbiana, con una mortalidad del 17\%. El índice de peritonitis de Mannheim es una herramienta clínica y un predictor de mortalidad, con alta sensibilidad y especificidad. Objetivo: Determinar si existe correlación entre el puntaje obtenido mediante el índice de peritonitis de Mannheim y la mortalidad. Método: Estudio retrospectivo, observacional, transversal, durante el periodo entre julio de 2013 y enero de 2014, en pacientes con diagnóstico de sepsis abdominal, en el Hospital General de México Dr. Eduardo Liceaga. Resultados: Se incluyeron 177 casos, 85 de sexo femenino y 92 de sexo masculino. La edad media fue de 43 años. El órgano causante de la sepsis abdominal más frecuente fue el apéndice. Fallecieron 18 pacientes (10.2\%). Se dividieron los casos, de acuerdo con el índice de Mannheim, en leves (< 25 puntos) y graves (> 26 puntos). De 27 pacientes graves, fallecieron 8 (29.6\%), mientras que de los 150 leves fallecieron 10 (6.7\%) (odds ratio: 5.895; intervalo de confianza del 95\%: 2.071-16.77; $p<0,05)$. Conclusión: El índice de Mannheim es un buen predictor de mortalidad para los pacientes con sepsis abdominal. El punto de corte óptimo es 20.

PALABRAS CLAVE: Sepsis abdominal. Mortalidad. Índice de peritonitis de Mannheim.

\begin{abstract}
Background: Abdominal sepsis represents the host's peritoneal inflammatory response to microbial invasion, which may be mild or severe, depending on the degree of contamination of the peritoneal cavity; with a reported mortality of $17 \%$. The Mannheim peritonitis index is a clinical tool and a predictor of mortality in patients with peritonitis, with high sensitivity and specificity. Objective: To determine if there is a correlation between the score obtained by the Mannheim peritonitis index, and mortality, in patients with abdominal sepsis. Method: A retrospective, observational, cross-sectional study, during the period between July 2013 and January 2014, in patients diagnosed with abdominal sepsis, attended at the General Hospital of Mexico Dr. Eduardo Liceaga. Results: We included 177 cases, 85 of the female sex and 92 of the male sex. The average age was 43 years. The most common organ responsible for abdominal sepsis was the appendix. Mortality of 18 cases (10.2\%). The cases were divided, according to the Mannheim index, in mild cases (< 25 points) and severe case (> 26 points). Of 27 severe cases, 8 (29.6\%) died, while of the 150 mild cases 10 (6.7\%) died (odds ratio: 5.895; 95\% confidence interval: 2.07116.77; $p$ < 0.05). Conclusions: The Mannheim scale is a good predictor of mortality for patients with abdominal sepsis. The optimal cut-off point is 20.
\end{abstract}

KEY WORDS: Abdominal sepsis. Mortality. Mannheim peritonitis index.

\author{
Correspondencia: \\ *Ansony Roger Godínez-Vidal \\ Balmis, 148 \\ Col. Doctores, Del. Cuauhtémoc \\ C.P. 06726, Ciudad de México, México \\ E-mail: ansony.rgv@gmail.com
}

Fecha de recepción: 04-03-2018

Fecha de aceptación: 04-05-2018

DOI: $10.24875 / C I R U .18000213$
Cir Cir. 2018;86:423-427

Contents available at PubMed www.cirugiaycirujanos.com 


\section{Antecedentes}

La peritonitis se define como la inflamación de la membrana serosa que delimita la cavidad abdominal y los órganos contenidos en ella. La infección de la cavidad peritoneal puede ocurrir por varias rutas: «espontánea», la cual se presenta por diseminación hematógena principalmente; la infección introducida a la cavidad peritoneal, observada predominantemente en pacientes con diálisis peritoneal y también susceptible de tratamiento únicamente con antibióticos, se denomina peritonitis primaria; la peritonitis terciaria se refiere a la infección peritoneal persistente o recurrente que suele presentarse en un paciente crítico con peritonitis secundaria, cuando las defensas del huésped y la terapia antimicrobiana han fracasado y se produce la sobreinfección por organismos resistentes, usualmente bacilos gramnegativos u hongos; la peritonitis secundaria se manifestará clínicamente por abdomen agudo, y su diagnóstico y tratamiento inicial serán necesariamente quirúrgicos; y la tercera ruta de infección de la cavidad peritoneal es por salida del contenido intestinal hacia ella, es polimicrobiana por naturaleza y se presenta como peritonitis generalizada (peritonitis secundaria) o como un absceso localizado'.

La sepsis abdominal es la respuesta sistémica a un proceso infeccioso inicialmente localizado, representa la respuesta inflamatoria peritoneal no específica del huésped ante la invasión microbiana y se manifiesta por datos sistémicos como fiebre, taquicardia, hipotensión, taquipnea, leucocitopenia o leucocitosis, y gasto cardiaco elevado; en los casos graves puede originar una alteración importante de la perfusión sistémica con falla orgánica múltiple².

\section{Mortalidad en la peritonitis secundaria}

La infección y la inflamación peritoneales generan una respuesta sistémica compleja que puede ser leve o grave, dependiendo del grado de contaminación de la cavidad peritoneal. Esta respuesta inflamatoria general origina cambios vasculares, celulares, inmunitarios y metabólicos que, si son persistentes, pueden provocar efectos isquémicos en diferentes lugares y desencadenar fallas orgánicas diversas (falla renal, hepática, pulmonar, cardiovascular, etc.). La mortalidad que se presenta por las causas principales de peritonitis secundaria no ha variado en las últimas dos décadas, en las que se ha reportado una mortalidad del $17 \%{ }^{3}$. Así mismo, el total de la mortalidad reportada para la apendicitis es del $1 \%$, y aumenta hasta el $5 \%$ cuando existe perforación, ocurriendo la mayoría de las muertes en pacientes de avanzada edad. Los factores pronósticos más importantes en cirugía colorrectal de urgencia son el estado preoperatorio, la edad avanzada y la presencia de peritonitis fecal, que juntos presentan una mortalidad de hasta el $60 \%{ }^{4}$.

\section{Predictores de mortalidad en cirugía}

Se han desarrollado diversos biomarcadores y escalas funcionales para predecir la morbilidad y la mortalidad en los pacientes quirúrgicos. Sin duda, el modelo de puntaje fisiológico de gravedad de la enfermedad más estudiado es Acute Physiology And Chronic Health Evaluation II (APACHE II) ${ }^{5}$, que se construye tomando el peor valor obtenido en las primeras 24 horas de admisión de 12 variables, con puntajes adicionados para edad y salud crónica, resultando en un puntaje total de 0 a 71 . La escala APACHE II ha sido validada en diversos estudios de pacientes quirúrgicos y no quirúrgicos en unidades de cuidados intensivos, presentando una media de puntaje de 9 a 15 entre los sobrevivientes y mostrando entre los que fallecieron una media de 15 a 25 puntos $^{6}$. Evaluando pacientes con peritonitis secundaria con puntajes entre 11 y 15 , APACHE II ha demostrado una sensibilidad del $100 \%$ y una especificidad del $73.8 \%$ para predecir mortalidad, y en el grupo de pacientes con puntajes entre 16 y 20 ha tenido una sensibilidad del $87.5 \%$ y una especificidad del $100 \%$.

\section{Índice de peritonitis de Mannheim}

El índice de peritonitis de Mannheim (IPM) (Tabla 1) es un predictor de mortalidad creado en Alemania por Wacha y Linder, el cual se basó en el resultado de 1253 pacientes con peritonitis, desarrollado por el análisis discriminativo de 17 factores de riesgo posibles, de los cuales ocho resultaron significativos para valor pronóstico. Permite una clasificación inmediata en dos grupos: baja y alta mortalidad. Los factores pronósticos que incluye son edad, sexo, falla orgánica, presencia de cáncer, origen colónico de la contaminación, peritonitis generalizada y características del fluido peritoneal (citrino, purulento o fecal), asignando distintos factores numéricos según sean resultados favorables 0 adversos. Se obtiene un puntaje de 0 a 47, con un punto de corte de 26, a partir del cual se estableció una mortalidad en los pacientes del $50 \%$, y en aquellos con puntajes menores de 26 tan solo del 1-3\%, con una sensibilidad del $95.9 \%$ y una especificidad del $80 \%$, un 
Tabla 1. Índice de peritonitis de Mannheim

\begin{tabular}{lc}
\hline Factor de riesgo & Valor si está presente \\
\hline Edad >50 años & 5 \\
Sexo femenino & 5 \\
Insuficiencia orgánica & 7 \\
Cáncer & 4 \\
Duración de la peritonitis > & 4 \\
24 h & \\
Sepsis de origen no colónico & 4 \\
Peritonitis generalizada & 4 \\
difusa & \\
Exudado claro & 0 \\
Citrino-purulento & 6 \\
Fecal & 12 \\
\hline
\end{tabular}

valor predictivo positivo del $98.9 \%$ y un valor predictivo negativo del $50 \%$. Debido a la gran diferencia entre el pronóstico de ambos grupos, otros han considerado establecer tres grupos pronósticos, con puntajes de corte entre ellos de menos de 21 puntos, de 21 a 29 puntos y mayor de 29 puntos, y una mortalidad del 2.3, el 22.5 y el 59.1 , respectivamente; la sensibilidad y la especificidad son similares a las establecidas para los grupos con punto de corte en 26 puntos $^{9-13}$.

La sepsis abdominal por peritonitis secundaria es un padecimiento frecuente, grave y con alta mortalidad potencial. Existen herramientas útiles para poder predecir la mortalidad del paciente con peritonitis, pero gran parte de ellas se basan en parámetros bioquímicos o emplean calculadoras o sistemas electrónicos especiales para su aplicación. El IPM es una herramienta clínica que se ha sugerido como predictor de mortalidad en pacientes con peritonitis, y ha resultado ser muy útil, sencillo, con alta sensibilidad y especificidad, que se integra con variables clínicas y hallazgos transoperatorios.

El objetivo de este estudio fue determinar si existe correlación entre el puntaje obtenido mediante el IPM y la mortalidad en pacientes con sepsis abdominal por peritonitis secundaria que requirieron manejo quirúrgico.

\section{Método}

Estudio retrospectivo, observacional, transversal, de pacientes atendidos en el Hospital General de México Dr. Eduardo Liceaga, durante el periodo entre julio de 2013 y enero de 2014, con diagnóstico de
Tabla 2. Clasificación por gravedad

\begin{tabular}{lcc}
\hline Indicador de gravedad & Leve & Grave \\
\hline APACHE II & $<14$ & $>15$ \\
Índice de peritonitis de & $<25$ & $>26$ \\
Mannheim & & \\
\hline
\end{tabular}

dolor abdominal agudo y que durante la cirugía se encontró sepsis abdominal por peritonitis secundaria.

\section{Resultados}

La muestra está integrada por 177 casos, 85 (48\%) del sexo femenino y $92(52 \%)$ del sexo masculino. La edad media fue de 43.38 años. El órgano causante de la sepsis abdominal más frecuente fue el apéndice, seguido de la vesícula biliar, el intestino delgado y el colon. Del total de la muestra, hubo un $10 \%$ de mortalidad.

Se dividieron los casos, de acuerdo con el IPM (Tabla 2), en leves (<25 puntos; 150 pacientes, $84.8 \%$ ) y graves ( $>26$ puntos; 27 pacientes, $15.2 \%$ ). Al dividir los casos de acuerdo con la gravedad evaluada mediante la escala APACHE II se encontró que 42 (23.7\%) fueron graves y 135 (76.3\%) fueron leves.

De los 27 pacientes graves por IPM fallecieron $8(29.6 \%)$, mientras que de los 150 pacientes leves por IPM fallecieron 10 (6.7\%), y al aplicar el estadístico ji al cuadrado a estas observaciones se obtiene un valor de $p<0,05$ (odds ratio: 5.895; intervalo de confianza del 95\%: 2.071-16.77).

Al analizar la curva ROC para IPM en su relación con la mortalidad obtuvimos un área bajo la curva de 0.84 , con un punto de corte de 20 , para una sensibilidad del $83 \%$ y una especificidad del $77 \%$ (Fig. 1).

\section{Discusión}

La sepsis abdominal por peritonitis secundaria es una patología con alta mortalidad e costos económicos elevados por la estancia hospitalaria prolongada y la terapéutica empleada en la unidad de terapia intensiva. La importancia del síndrome de respuesta inflamatoria sistémica secundario a la patología abdominal puede mostrar evidencia de disfunción orgánica limitada a uno o dos órganos, o desarrollar una respuesta inflamatoria exagerada que rápidamente progresa a la muerte, pocos días después del trauma inicial, secundaria a insuficiencia orgánica múltiple ${ }^{14}$. La mortalidad con disfunción 
orgánica múltiple es proporcional al número de sistemas implicados y varía del 30\% con insuficiencia de órgano único al $100 \%$ con cinco órganos afectados $^{15}$.

Diversos estudios han ideado sistemas de puntuación para predecir la morbimortalidad en la cirugía de urgencia. La puntuación APACHE II se utiliza en la unidad de cuidados intensivos y requiere 12 parámetros fisiológicos durante un período de 24 horas para calcularlo, por lo que no es posible utilizarlo en la cirugía de urgencia. En los pacientes con patología quirúrgica por sepsis abdominal, además de la puntuación APACHE II se han desarrollado otros sistemas, como el IPM, la puntuación de falla orgánica múltiple y la puntuación de sepsis ${ }^{16}$. En estudios comparativos, la escala APACHE II y el IPM ofrecen una mejor predicción de la mortalidad en los pacientes con sepsis abdominal. La combinación de IPM y APACHE II mejora la especificidad, pero son utilizadas principalmente al evaluar los resultados de pacientes quirúrgicos ingresados en unidades de cuidados intensivos después de la cirugía. El IPM es simple de calcular y útil en la predicción de la mortalidad, con una sensibilidad del $83 \%$ y una especificidad del $77 \%$. En nuestro estudio analizamos el IPM tomando un punto de corte estandarizado internacional, y nuestro resultado corrobora que el IPM se correlaciona de forma directa con la mortalidad de nuestra muestra. Al obtener este resultado, realizamos una curva ROC para obtener un mejor punto de discriminación para determinar la mortalidad, y dicha curva nos reporta un puntaje de 20 puntos con un área bajo la curva mayor de 80 , una sensibilidad del $83 \%$ y una especificidad del $77 \%$. Así, podemos asumir que a mayor puntaje de IPM, mayor mortalidad (Fig. 2). Este resultado se tomará en cuenta en futuros estudios prospectivos para estandarizar nuestro punto de corte.

Actualmente, la peritonitis secundaria es frecuente en cirugía general. Después de la laparotomía inicial surge la duda de una segunda intervención por la evolución posquirúrgica del paciente. Existen parámetros, como la frecuencia cardiaca, la hemoglobina, la temperatura corporal, la presencia de evacuaciones, la extensión de la contaminación en la primera cirugía y la necesidad de inotrópicos, que aumentan el riego de una segunda intervención. Sin embargo, aún no se ha diseñado una escala ideal de mortalidad que sea precisa, fácil de calcular y reproducible, tanto en el preoperatorio como en el posoperatorio, que permita evaluar la respuesta al manejo quirúrgico empleado.

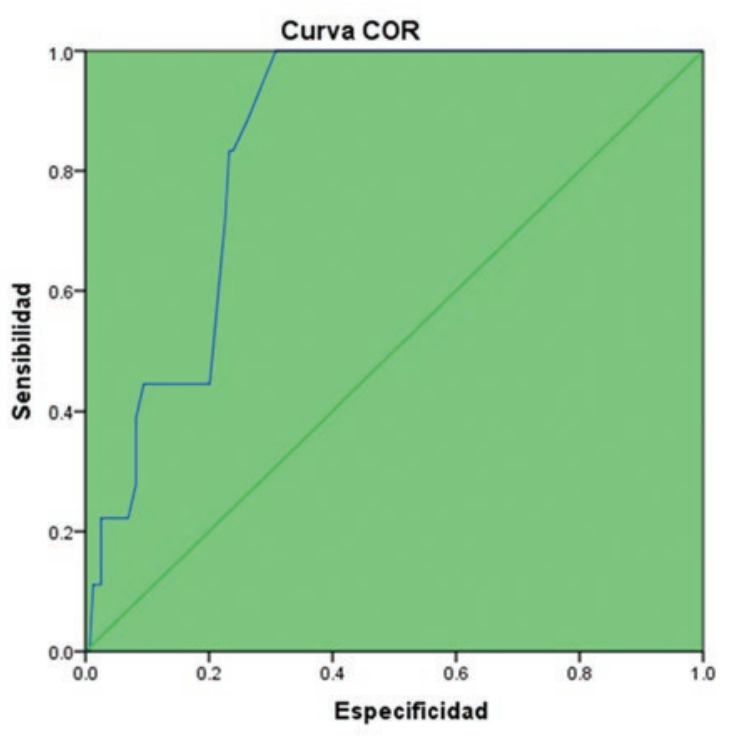

Los segmentos de diagonal se generan mediante empates.

Figura 1. Área bajo la curva de 0.84 , con un punto de corte de 20 , para una sensibilidad del $83 \%$ y una especificidad del $77 \%$.

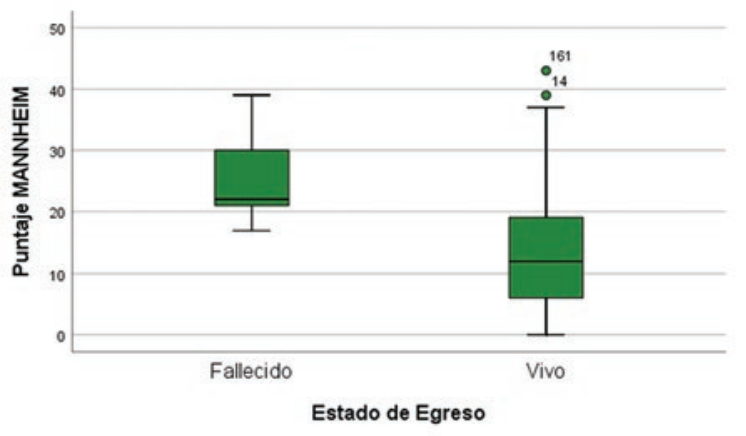

Figura 2. A mayor puntaje del índice de Mannheim, mayor mortalidad.

\section{Conclusión}

El IPM es un buen predictor de mortalidad para los pacientes con sepsis abdominal por peritonitis secundaria. En nuestra población, el punto de corte óptimo para determinar la gravedad de los pacientes sépticos abdominales mediante el IPM es de 20 puntos.

\section{Financiamiento}

No se obtuvo ningún tipo de financiamiento por parte de alguna empresa.

\section{Conflicto de intereses}

No existe ningún conflicto de intereses. 


\section{Bibliografía}

1. Townsend Jr CM, Beauchamp RD, Evers BM, Mattox KL. Sabiston Textbook of Surgery. $18^{\text {th }}$ ed. Philadelphia: WB Saunders. 2008.

2. Carrillo-Esper R, Carrillo-Córdova JR, Carrillo-Córdova LD. Estudio epidemiológico de la sepsis en unidades de terapia intensiva mexicanas. Cir Cir. 2009:77(4)

3. Pulido Cejudo A. FACS. Tratamiento médico en sepsis abdominal. Infección en cirugía. Cirujano General. 2011;33(Supl 1).

4. Dombrovskiy VY, Martin AA, Sunderram J, Paz HL. Rapid increase in hospitalization and mortality rates for severe sepsis in the United States: a trend analysis from 1993 to 2003. Crit Care Med. 2007;35:1244.

5. Sánchez Velázquez LD, Reyes Sánchez ME, D’Ector Lira DM, Bonilla Rivera LC, González González A, Magdaleno Padilla ML, et al. Discriminación y calibración de cuatro escalas de calificación del síndrome de disfunción orgánica múltiple. Estudio multicéntrico. Rev Asoc Mex Med Crit y Ter Int 2001;15(3):80-86

6. Pardo Cabello AJ, Bermudo Conde S, Manzano Gamero MV. Prevalencia y factores asociados a desnutrición entre pacientes ingresados en un hospital de media-larga estancia. Nutr Hosp. 2011;26:369-75.

7. John MA, Bohnen MD, Mustard RA, Oxholm SE, Schouten BD. APACHE II Score and Abdominal Sepsis A Prospective Study, RN Arch Surg. 1988;123(2):225-22.

8. Vincent $\mathrm{JL}$ and Moreno R. Clinical review: Scoring systems in the critically ill. Critical Care 2010, 14:207.
9. Mesejo Arizmendi A, Blasco Cortés ML, Gabaldón Coronado J. Manual básico de nutrición clínica y dietética. Hospital Clínico Universitario de Valencia; 2000. p. 17-8.

10. Kreger BE, Craven DE, McCabe WR. Gram-negative bacteremia. IV. Re-evaluation of clinical features and treatment in 612 patients. Am J Med. 1980;68:344

11. Planas Vilá M, Montejo JC. Metodología aplicada en la valoración del estado nutricional. Libro blanco de la desnutrición clínica en España. Fundación Española de la Nutrición (FEN). 2013.

12. Bauer J, Capra S. Comparison of a malnutrition-screening tool with subjective global assessment in hospitalized patients with cancer: sensitivity and specificity. Asia Pac J Clin Nutr. 2003:12:257-60.

13. Sharma R, Ranjan V, Jain S, Joshi T, Tyagi A, Chaphekar R. A prospective study evaluating utility of Mannheim peritonitis index in predicting prognosis of perforation peritonitis. J Nat Sci Biol Med. 2015;6(Suppl 1):49-S52.

14. Sharma R, Ranjan V, Jain S, Joshi T, Tyagi A, Chaphekar R. A prospective study evaluating utility of Mannheim peritonitis index in predicting prognosis of perforation peritonitis. J Nat Sci Biol Med. 2015;6(Suppl1):S49-52.

15. Salamone G, Licari L, Falco N, Augello G, Tutino R, Campanella S, et al. Mannheim Peritonitis Index (MPI) and elderly population: prognostic evaluation in acute secondary peritonitis. G Chir. 2016;37:243-9.

16. Muralidhar VA, Madhu CP, Sudhir S, Srinivasarangan M. Efficacy of Mannheim Peritonitis Index (MPI) score in patients with secondary peritonitis. J Clin Diagn Res. 2014;8:NC01-3. 